\title{
A Microwave Method for Complex Permittivity Extraction of Thin Materials
}

\author{
Nawfal Jebbor \\ Spectrometry Laboratory of Materials and Archeomaterials (LASMAR) Faculty of Sciences, Moulay Ismail \\ University, Meknes - Morocco \\ jebbor.nawfal@gmail.com \\ Seddik Bri \\ Electrical Engineering Department, Technology High School (ESTM), Moulay Ismail University, Meknes- \\ Morocco \\ briseddik@gmail.com
}

\begin{abstract}
An improved microwave method to extract the complex permittivity of solid and liquid materials filled in a short-circuited waveguide is developed. The method determines accurately the dielectric constant of thin and moderate thick samples. It eliminates the problems arising from any position offset of the dielectric slab in transmission / reflection methods. The proposed method is iterative and the initial value is calculated by using the 7th approximation order of trigonometric terms in the exact reflection coefficient equation. This approach is applied to the simulated data of low loss and dissipative materials in limited frequency band.
\end{abstract}

Index Terms - Complex permittivity, Rectangular waveguide, Thin materials, $\mathrm{X}$ band.

\section{INTRODUCTION}

In the microwave frequencies, the electrical and magnetic properties of the dielectric medium are strongly influenced by the frequency. Given that the increased use of nanocomposite materials, there is a growing industrial demand for dielectric characterization of these new materials, starting from the designing phase through the control process via the production stage. Therefore, the knowledge of the constituent concentrations (resin binder, carbon black filled epoxy polymer), provides the ability to size the desired dielectric properties of the composite materials. However, there is a growing demand for accurate microwave techniques to characterize solids, liquids and granular materials over broadband frequencies and at different temperatures [1], [2], [3], [4].

Various microwave methods are proposed in literature with their advantages and drawbacks. Although the resonant techniques [5], [6], [7] are more accurate than the nonresonant techniques [8], [9], they require a meticulous sample preparation of the material under test. Practically, this is no longer appreciated. The nonresonant methods are widely used to characterize different kinds of materials over a broad frequency band. However, they require a less sample preparation compared to resonant methods. Due to its simplicity, the nonresonant waveguide transmission / reflection method is presently the most widely used broadband measurement technique; it is also applied for measuring the dielectric constant of thin materials [10], [11], [12], [13].

In this regard and in accordance with the transmission and/or reflection techniques, K. Sarabandi 
and his co-worker F. T. Ulaby have based, in their paper [12], on measuring the reflection coefficient $\Gamma$ of a thin sample filled in a rectangular waveguide. The exact expression of $\Gamma$ is simplified in the case of thin materials and they obtain an explicit expression of the first and second orders of the relative dielectric constant $\varepsilon_{\mathrm{r}}$. But a small offset positioning of the slab inside the waveguide will generate enormous errors in the inversion of $\varepsilon_{\mathrm{r}}$ from the measured $\Gamma$. On the other hand, in the article of B. K. Chung [13], the relative permittivity determination is performed from the measured transmission coefficient $T$. Chung obtains an explicit expression of $\varepsilon_{\mathrm{r}}$ via a second order approximation. Moreover, his method eliminates to a certain level of achievement the offset positioning problem encountered in Sarabandi technique. In this article, we present a method to characterize thin dielectric materials by measuring the reflection coefficient of a short-circuited rectangular waveguide shown in Fig. 1.

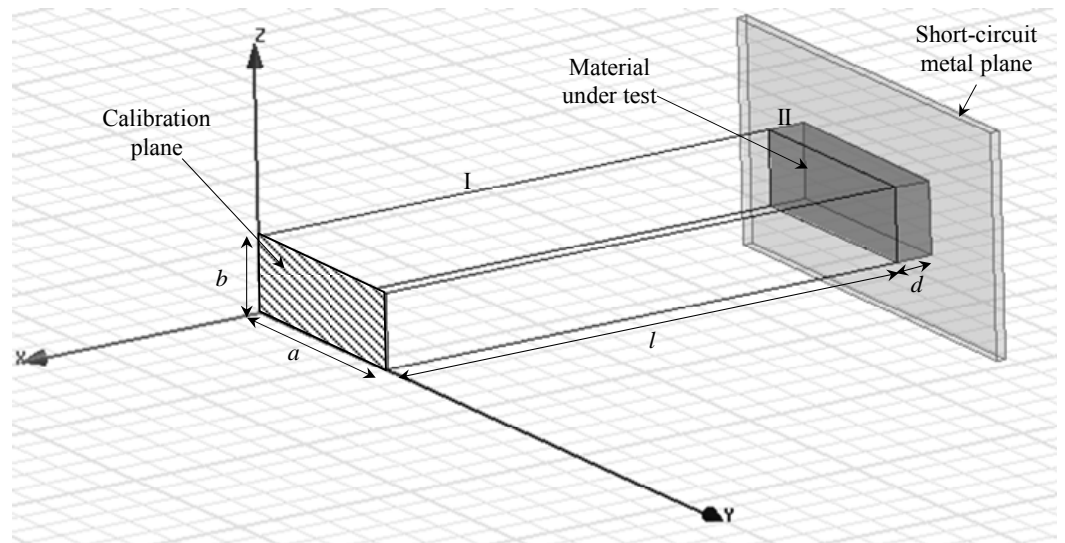

Fig. 1. Illustration of the problem. Rectangular waveguide with a dielectric slab material occupying region II (between $x=-l$ and $x=-(l+d)$ ).

The slab under test is in direct contact with the metal plane of short-circuit without air gap and the offset positioning problem is definitively eliminated. The trigonometric terms of the exact expression of $\Gamma$ are linearized in the case of thin materials and one complex relative permittivity value is extracted. This technique uses higher order approximations and the accuracies are compared to the true value of dielectric slab and to Sarabandi / Chung approximations. In one objective to get a relative permittivity as accurately as possible, the exact expression of $\Gamma$ is solved by NewtonRaphson method with an initial guess of the iterative process is $\varepsilon_{\mathrm{r}}$ which is a solution of the approximated equation of $\Gamma$. However, the initial value problem encountered in iterative techniques is thus overcome. Once the initial guess is known, one can proceed to another traditional measurement [14] for the case where it is possible to fill the entire sample holder with the material under test (liquid or solid). By using the electromagnetic 3D-simulation software Ansoft HFSS, the studied structure of Fig. 1 is implemented and the relative dielectric constant of Teflon, wood and distilled water are extracted via the proposed method. The experimental setup can help immensely to characterize new nanocomposite materials mainly in microwave frequencies and in a wide range of temperatures [1], [2], [4]. 


\section{COMPLEX PERMITTIVITY THEORY DEVELOPMENT}

Consider a short-circuited rectangular waveguide of dimensions $(a \times b)$ containing a thin slab placed in a plane orthogonal to the propagation direction $O x$ and in direct contact with the short-circuit plane as shown in Fig. 1. In the analysis, the sample is assumed isotropic, symmetric, homogeneous and non-magnetic $\left(\mu_{r}=1\right)$. The dielectric slab of thickness $d$ is located at $x=-l . l$ is the distance between the calibration plane and the air-sample interface. In an objective to seek a relationship between the reflection coefficient $S_{11}=\Gamma$ and the relative permittivity $\varepsilon_{\mathrm{r}}=\varepsilon_{\mathrm{r}}{ }^{\prime}-j \varepsilon_{\mathrm{r}}{ }^{\prime}$ of the dielectric slab material, we shall develop expressions of electric and magnetic fields in regions I and II from their potentials $\overrightarrow{\mathrm{A}}$ and $\overrightarrow{\mathrm{F}}$ such as [10], [11], [15]:

$$
\begin{aligned}
& \overrightarrow{\mathrm{E}}=-j \omega \overrightarrow{\mathrm{A}}-\mathrm{j} \frac{1}{\omega \mu_{0} \varepsilon_{0} \varepsilon_{\mathrm{r}}} \nabla(\nabla \cdot \overrightarrow{\mathrm{A}})-\frac{1}{\varepsilon_{0} \varepsilon_{\mathrm{r}}} \nabla \times \overrightarrow{\mathrm{F}} \\
& \overrightarrow{\mathrm{H}}=\frac{1}{\mu_{0}} \nabla \times \overrightarrow{\mathrm{A}}-\mathrm{j} \omega \overrightarrow{\mathrm{F}}-\mathrm{j} \frac{1}{\omega \mu_{0} \varepsilon_{0} \varepsilon_{\mathrm{r}}} \nabla(\nabla \cdot \overrightarrow{\mathrm{F}})
\end{aligned}
$$

Where $\varepsilon_{0}$ and $\mu_{0}$ are the permittivity and permeability of free-space respectively. Assuming that the rectangular waveguide operates in the dominant mode $\mathrm{TE}_{10}$, we have $\overrightarrow{\mathrm{A}}=\overrightarrow{0}$ and $\partial \mathrm{F}_{\mathrm{x}} / \partial \mathrm{y}=0$ [15]. Then, the electric vector potential can be written for regions I and II as:

$$
\begin{array}{cc}
\mathrm{F}_{\mathrm{x}}^{(\mathrm{I})}(\mathrm{x}, \mathrm{z})=\cos \left(\frac{\pi \mathrm{z}}{\mathrm{a}}\right)\left[\mathrm{C}_{1} \mathrm{e}^{\gamma_{0} \mathrm{x}}+\mathrm{C}_{2} \mathrm{e}^{-\gamma_{0} \mathrm{x}}\right] & -1 \leq \mathrm{x} \leq 0 \\
\mathrm{~F}_{\mathrm{x}}^{(\mathrm{II})}(\mathrm{x}, \mathrm{z})=\cos \left(\frac{\pi \mathrm{z}}{\mathrm{a}}\right)\left[\mathrm{C}_{3} \mathrm{e}^{\gamma \mathrm{x}}+\mathrm{C}_{4} \mathrm{e}^{-\gamma \mathrm{x}}\right] & -1-\mathrm{d} \leq \mathrm{x} \leq-1 \\
\gamma_{0}=\mathrm{j} 2 \pi / \lambda_{0} \sqrt{1-\lambda_{0}^{2} / \lambda_{\mathrm{c}}^{2}}, \quad \gamma=\mathrm{j} 2 \pi / \lambda_{0} \sqrt{\varepsilon_{\mathrm{r}}-\lambda_{0}^{2} / \lambda_{\mathrm{c}}^{2}}
\end{array}
$$

Where

$\lambda_{0}=c / f$ and $\lambda_{\mathrm{c}}=c / f_{\mathrm{c}}$ correspond to the free space and cut-off wavelengths; and $f, f_{\mathrm{c}}$ and $c$ are the operating and cut-off frequencies and the speed of light in vacuum, respectively. The time factor $\mathrm{e}^{\mathrm{j} \omega t}$ was assumed and suppressed. The unknown coefficients $\mathrm{C}_{1}, \mathrm{C}_{2}, \mathrm{C}_{3}$ and $\mathrm{C}_{4}$ can be obtained by applying the appropriate boundary conditions at $x=-l$ and $x=-(l+d) . \mathrm{C}_{1}$ and $\mathrm{C}_{2}$ represent the magnitudes of the incident and reflected waves in region $\mathrm{I} ; \mathrm{C}_{3}$ and $\mathrm{C}_{4}$ represent the magnitudes of the incident and the reflected waves in region II.

The complex reflection coefficient $S_{11}$ is found to be:

$$
\mathrm{S}_{11}=\Gamma=\frac{\mathrm{C}_{2}}{\mathrm{C}_{1}}=\mathrm{R}^{2} \frac{\tanh (\gamma \mathrm{d})-\gamma / \gamma_{0}}{\tanh (\gamma \mathrm{d})+\gamma / \gamma_{0}}
$$

Where $\mathrm{R}=\mathrm{e}^{-\gamma_{0} l}$ is the transformation factor from the air-sample interface to calibration plane. For thin materials case, $(\gamma \mathrm{d})$ is small, and the hyperbolic tangent terms of (6) can be approximated by:

$$
\tanh (\gamma \mathrm{d}) \cong(\gamma \mathrm{d})-\frac{1}{3}(\gamma \mathrm{d})^{3}+\frac{2}{15}(\gamma \mathrm{d})^{5}-\frac{17}{315}(\gamma \mathrm{d})^{7}
$$


Multiple constant propagation values $\gamma_{i}$ can satisfy the approximated expression of (6); noted (6-7). High accurate results can be attained by increasing the degree of the approximation in (6-7), and by using "solve" function of MATLAB, we can find $\gamma_{\mathrm{i}}$ solutions of (6-7). The choice of the unique $\varepsilon_{\mathrm{r}}$ will build on the solution $\gamma=\alpha+j \beta$ of (6-7) that represents the best propagated mode ( $\gamma$ corresponds to the smaller $\alpha$-value of the solutions $\gamma_{i}$ with $\beta$ is positive).

\section{SENSITIVITY TO SAMPLE THICKNESS}

In this section, the objective is focused on the accuracy of the approximated expression (6-7) which depends greatly on the magnitude of $(\gamma d)$. To analyze the sensitivity, we compute the exact reflection coefficient $S_{11}$ using equation (6) and apply it in (6-7) to find the approximate solution $\varepsilon_{\mathrm{r}}$. The solutions of (6-7) that correspond to the third, the fifth and the seventh approximation orders of tanh terms are compared with Sarabandi and Chung techniques for various cases. For example, Fig. 2 and 3 demonstrate the dependency of $\varepsilon_{\mathrm{r}}$ of a low loss sample $\left(\varepsilon_{\mathrm{r}}=2-j 0.05\right)$ and a lossy sample $\left(\varepsilon_{\mathrm{r}}=20-j 10\right)$ versus the dielectric slab lengths.

The test parameters are $f=10 \mathrm{GHz}$ and $f_{\mathrm{c}}=6.555 \mathrm{GHz}$ (a WR-90 waveguide with $\mathrm{a}=22.86 \mathrm{~mm}$ and $\mathrm{b}=10.16 \mathrm{~mm}$ is assumed). In Fig. 2, we observe that the $5^{\text {th }}$ approximation order ( $5^{\text {th }}$ A.O.) provides values that are within $0.07 \%$ of $\varepsilon_{\mathrm{r}}$ ' and within $0.2 \%$ of $\varepsilon_{\mathrm{r}}$ " for $d \leq 1 \mathrm{~mm}$. However, the $7^{\text {th }}$ approximation order $\left(7^{\text {th }}\right.$ A.O.) provides more accurate values of $\varepsilon_{\mathrm{r}}$ ' and $\varepsilon_{\mathrm{r}}$ " for low loss materials than the $5^{\text {th }}$ A.O. In contrast, and for lossy materials (e.g. $\varepsilon_{\mathrm{r}}=20-j 10$ ), the results reported in Fig. 3 show that only the $7^{\text {th }}$ A.O. leads to values within $1 \%$ of $\varepsilon_{\mathrm{r}}{ }^{\prime}$ and within $1.6 \%$ of $\varepsilon_{\mathrm{r}}$ " for $d \leq 1 \mathrm{~mm}$. The $3^{\text {rd }}$ and the $5^{\text {th }}$ A.O.; and Sarabandi/Chung techniques are inaccurate and inadequate for approximating complex value of $\varepsilon_{\mathrm{r}}$ for lossy materials that the thickness is more than $0.25 \mathrm{~mm}$. It is seen from Fig. 2 and 3 that while the Sarabandi reflection method [12] and the Chung transmission method [13] extract roughly similar results for $\varepsilon_{\mathrm{r}}$. Our proposed method determines much better $\varepsilon_{\mathrm{r}}$ values for thin slab lengths.

A summary of relative accuracies of the $5^{\text {th }}$ and $7^{\text {th }}$ A.O. are presented in Table I for slab thicknesses $d$ equal to $5 \%, 10 \%$ and $20 \%$ of $\lambda_{0} / \sqrt{|\varepsilon|}$. The data of the table are the maximun realtive errors defined as : $\mathrm{e}^{\prime}=\operatorname{Max}\left(\left|\left(\varepsilon_{\mathrm{r}}^{\prime}-\varepsilon^{\prime}\right) / \varepsilon^{\prime}\right|\right) \times 100$. Where $\varepsilon_{\mathrm{r}}{ }^{\prime}$ and $\varepsilon^{\prime}$ are the real parts of the $\varepsilon_{\mathrm{r}}$-approximate solution of (6-7) and the exact value of the dielectric slab respectively. All entries are computed considering three virtual materials $5-j 5,5-j 0.5$ and 5-j0.05 at $f=10 \mathrm{GHz}$. In Fig. 4, we present the sensitivity of the $7^{\text {th }}$ A.O. of expression (6-7) to the thickness $d$ as a function of three frequency points 8.2 GHz, $10 \mathrm{GHz}$ and $12.4 \mathrm{GHz}$. However, the method is well suited for very low loss materials, and estimating $\varepsilon_{\mathrm{r}}$ ' can be extended to $6 \mathrm{~mm}$ sample thickness at the first frequency point $(f=8.2 \mathrm{GHz})$. This can be very useful when we do not know the initial value for iterative calculation techniques to characterize lossless materials. 


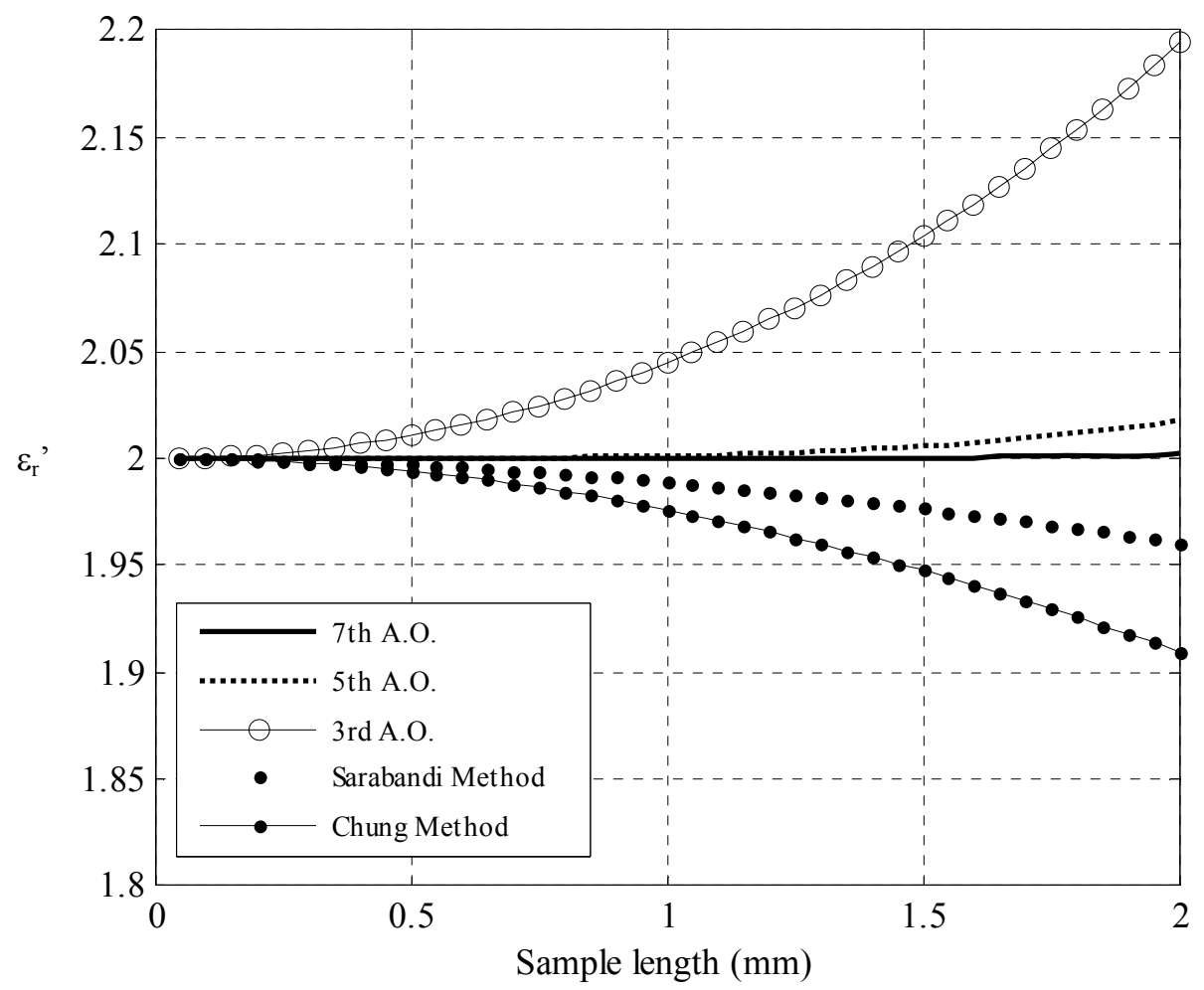

(a)

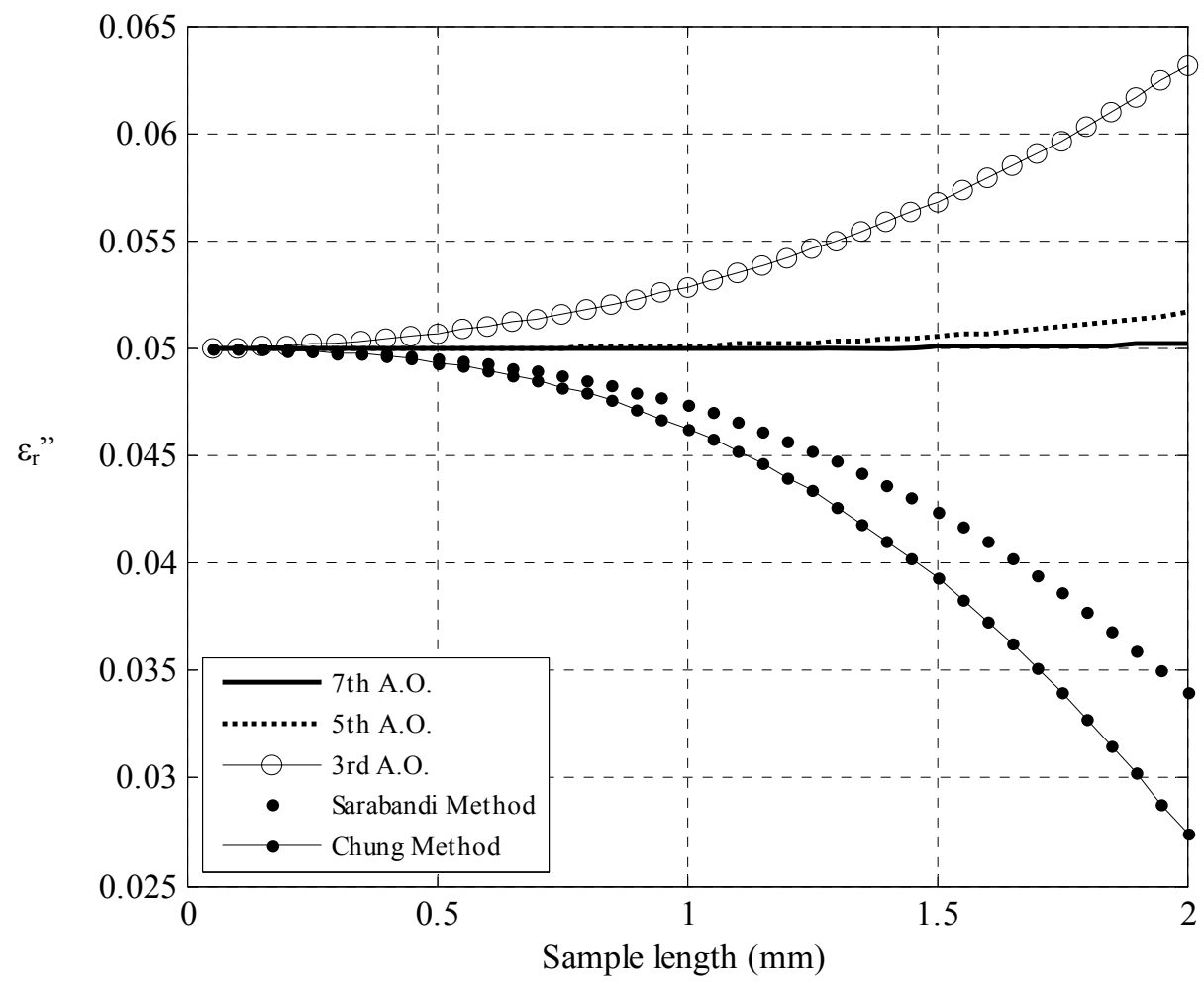

(b)

Fig. 2. Dependency of the relative permittivity ((a) real part, and (b) imaginary part) of a lowloss material $(\varepsilon=2-j 0.05)$ versus sample length. The $3^{\text {rd }}, 5^{\text {th }}$ and $7^{\text {th }}$ approximation orders (A.O.) of the proposed method are comapared to Sarabandireflection method [12] and Chung-transmission method [13]. 


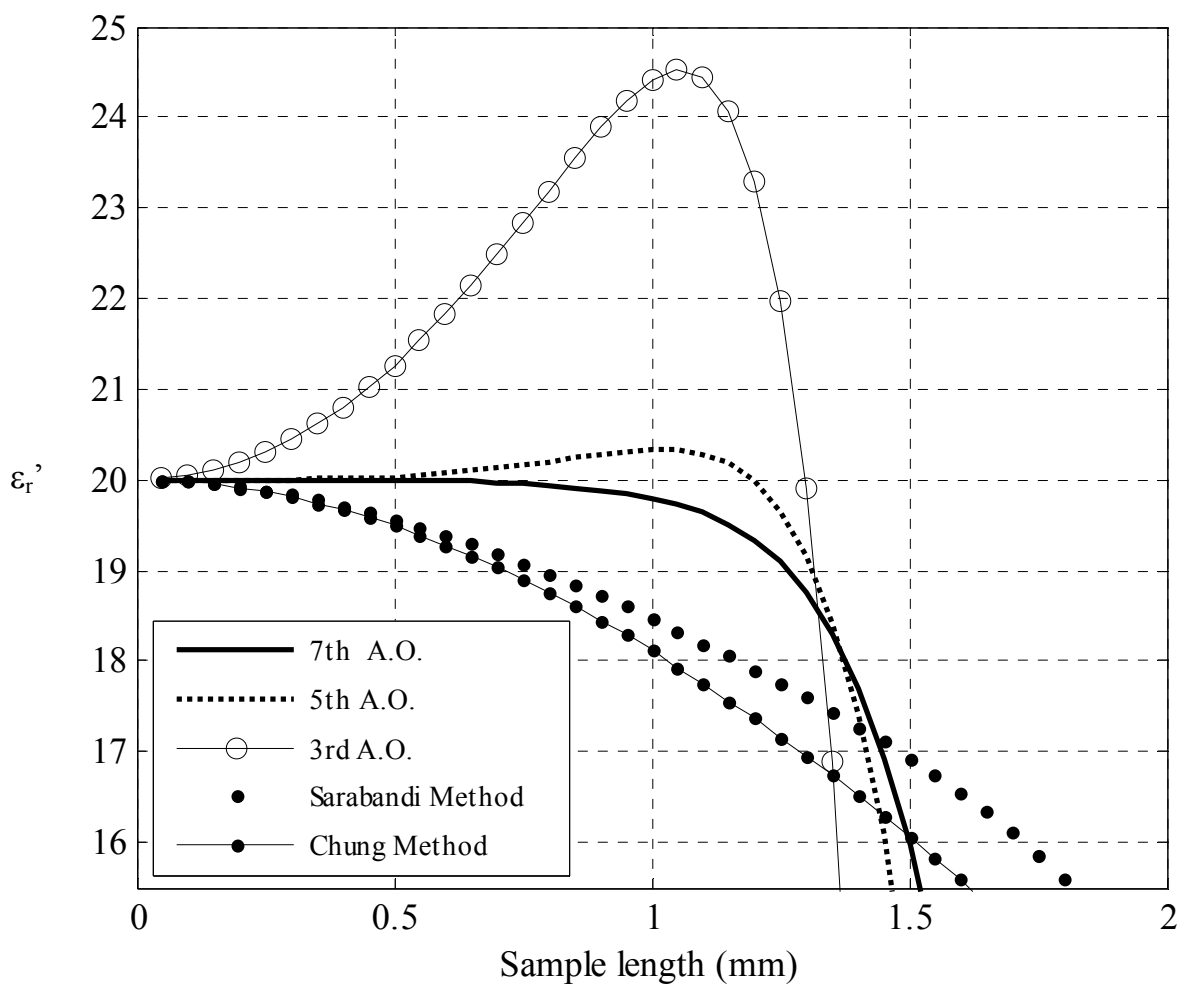

(a)

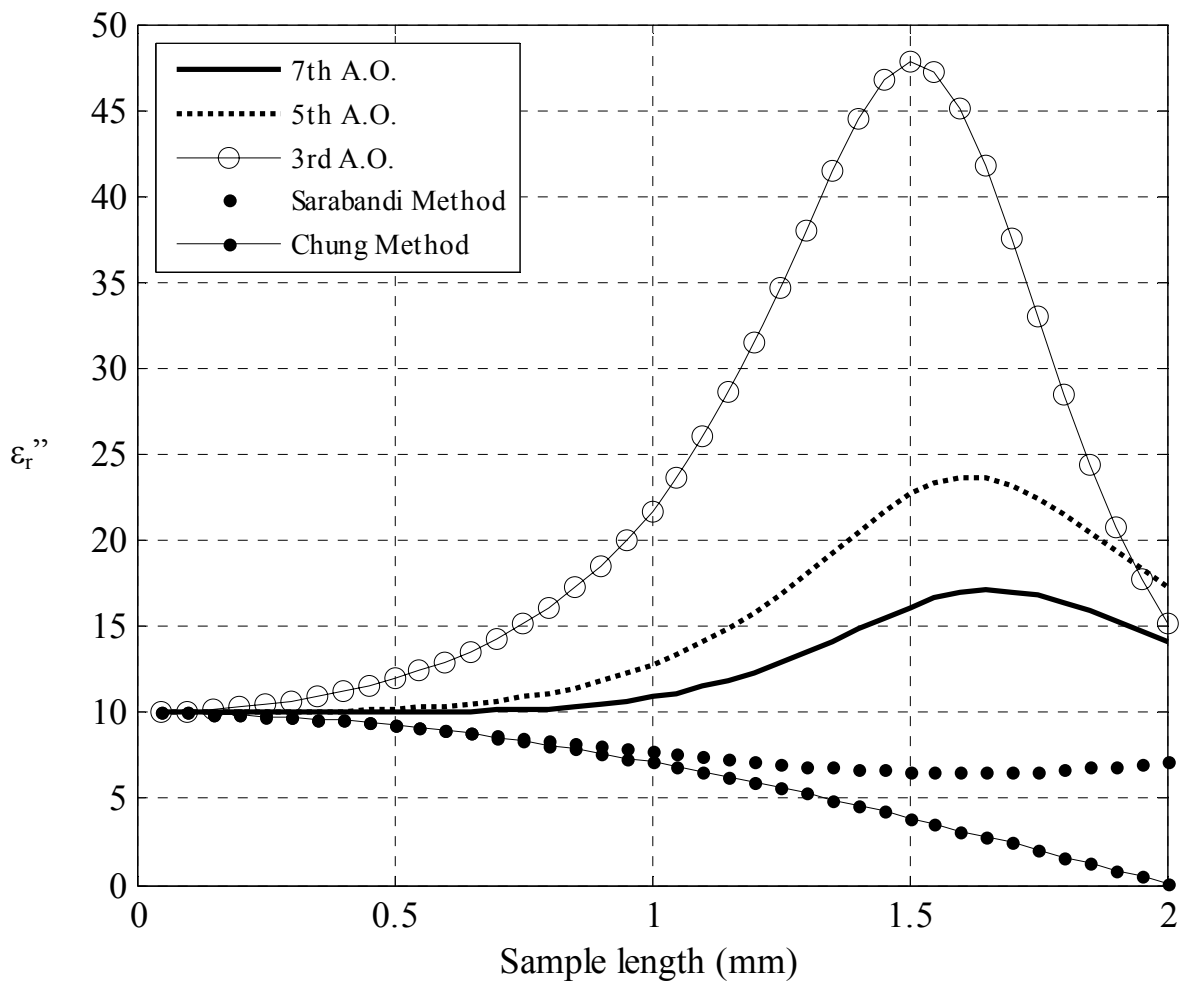

(b)

Fig. 3. Dependency of the relative permittivity ((a) real part, and (b) imaginary part) of a lossy material ( $\varepsilon=20-j 10)$ versus sample length. The $3^{\text {rd }}, 5^{\text {th }}$ and $7^{\text {th }}$ approximation orders (A.O.) of the proposed method are compared to Sarabandi-reflection method [12] and Chung-transmission method [13]. 
TABLE I. MAXIMUM RELATIVE ERRORS IN PERCENT OF $\varepsilon_{\mathrm{r}}$ ASSOCIATED WITH THE $5^{\text {TH }}$ AND THE $7^{\text {TH }}$ APPROXIMATION ORDERS.

\begin{tabular}{|c|c|c|c|c|c|c|c|c|c|}
\hline \multirow{3}{*}{$\begin{array}{c}\text { Maximum } \\
\text { relative } \\
\text { error in } \\
\%\end{array}$} & \multicolumn{3}{|c|}{$\mathrm{d} \sqrt{|\varepsilon|} / \lambda_{0}=0.05$} & \multicolumn{3}{|c|}{$\mathrm{d} \sqrt{|\varepsilon|} / \lambda_{0}=0.1$} & \multicolumn{3}{|c|}{$\mathrm{d} \sqrt{|\varepsilon|} / \lambda_{0}=0.2$} \\
\hline & \multicolumn{3}{|c|}{$\varepsilon^{\prime \prime} / \varepsilon^{\prime}$} & \multicolumn{3}{|c|}{$\varepsilon^{\prime \prime} / \varepsilon^{\prime}$} & \multicolumn{3}{|c|}{$\varepsilon^{\prime \prime} / \varepsilon^{\prime}$} \\
\hline & 0.01 & 0.1 & 1 & 0.01 & 0.1 & 1 & 0.01 & 0.1 & 1 \\
\hline $\mathbf{e}_{\text {5th A.o. }}$ & 0.12 & 0.16 & 0.146 & 1.56 & 1.51 & 1.98 & 32.87 & 30.52 & 32.71 \\
\hline $\mathrm{e}{ }_{5 \text { th A.o. }}$ & 0.40 & 0.38 & 0.116 & 5.00 & 5.02 & 1.7 & 132.00 & 128.68 & 17.62 \\
\hline $\mathbf{e}^{\prime}{ }_{7 \text { th }}$ A.O. & 0.00 & 0.004 & 0.008 & 0.21 & 0.196 & 0.38 & 13.45 & 12.25 & 18.47 \\
\hline $\mathrm{e}{ }_{7 \text { th A.O. }}$ & 0.00 & 0.02 & 0.002 & 0.80 & 0.88 & 0.026 & 60.00 & 60.04 & 0.80 \\
\hline
\end{tabular}

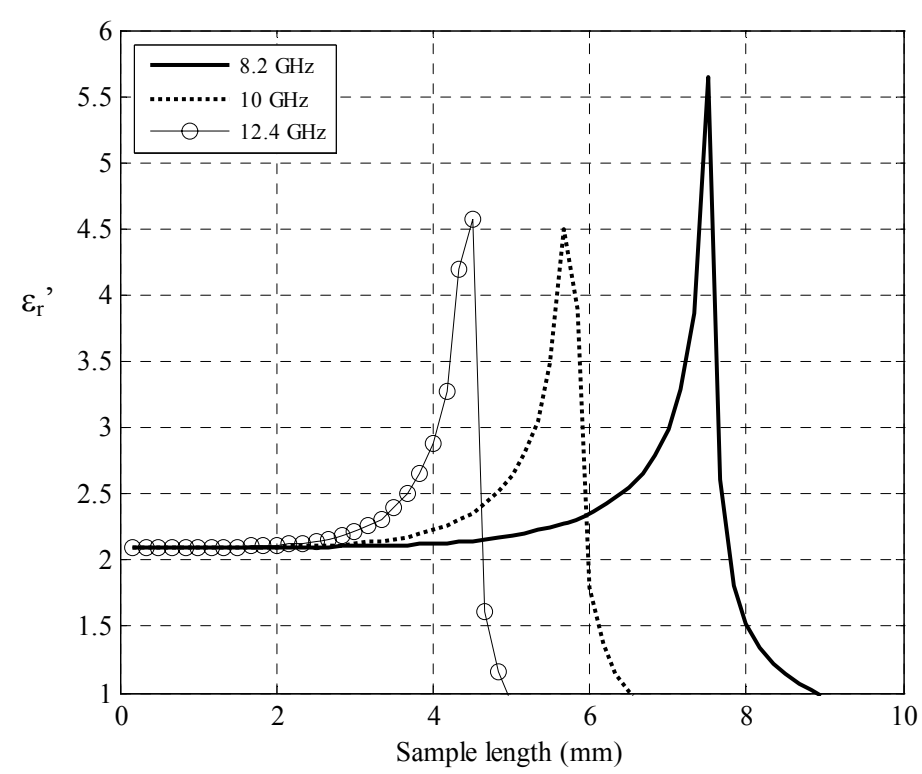

Fig. 4. Dependency of $\varepsilon_{\mathrm{r}}$ ' of a lossless material $(\varepsilon=2.1-j 0.0016$; Teflon $)$ versus sample length. The $7^{\text {th }}$ A.O. solutions are computed for three frequency points:

$8.2 \mathrm{GHz}, 10 \mathrm{GHz}$ and $12.4 \mathrm{GHz}$.

\section{HFSS SOFTWARE SIMULATIONS}

HFSS is a high performance full wave electromagnetic field simulator for 3D modeling structures. HFSS can be used to calculate scattering parameters, resonant frequency and fields. In industry, HFSS is the tool for high productivity research and development. HFSS is based on the finite element method which computes the electromagnetic fields in the frequency domain by solving Maxwell's equations locally. The 3D structure of Fig. 1 is designed and electromagnetic properties have been assigned to each object:

- Microwave source is assigned to the calibration plane.

- Perfect electrically conducting (PEC) walls are assigned to all external surfaces except source.

- Air electromagnetic properties are assigned the inner volume of region I.

- Electromagnetic properties of the material under test are assigned to the inner volume of region II. 
The HFSS simulations are performed for 40 frequency points from $8.2 \mathrm{GHz}$ to $12.4 \mathrm{GHz}$. Then, $\mathrm{S}_{11}$ parameter data file is exported and incorporated into MATLAB program in order to recover the software material properties of the material under test via the proposed method.

\section{NUMERICAL VALIDATION}

The waveguide structure of the Fig. 1 has been simulated by the Ansoft HFSS software. The reflection coefficient $S_{11}$ is computed at the calibration plane for $40 \mathrm{X}$-band frequency points. The numerical results of $S_{11}$ are thereafter incorporated into MATLAB program to retrieve the starting relative permittivity. The flowchart of the Fig. 5 describes the algorithm of MATLAB program.

The solving iterative process of equation (6) is started with an initial value $\varepsilon_{\mathrm{r} 0}$ which is a solution of the $7^{\text {th }}$ A.O. of equation (6-7). The starting $S_{11}$ corresponds to the lowest frequency point. This choice is based on the results of Fig. 4 and helps to extract the dielectric constant of materials as thick as possible. $\varepsilon_{\mathrm{r} 0}$ is the initial value of the first loop and $\varepsilon_{\mathrm{r} 0}$ of the next loop is equal to $\varepsilon_{\mathrm{r}}$ that is the solution of the previous iteration. We note that the equation (6) is resolved iteratively by the Newton-Raphson method. We check the proposed method for Teflon, wood and distilled water. For liquid characterization case, the experimental setup of Fig. 1 is positioned vertically. But for this paper, we will deal only the numerical validation of the proposed approach. The flowchart of Fig. 5 summarizes all steps in totality including the HFSS simulation results and the iterative calculation by MATLAB.

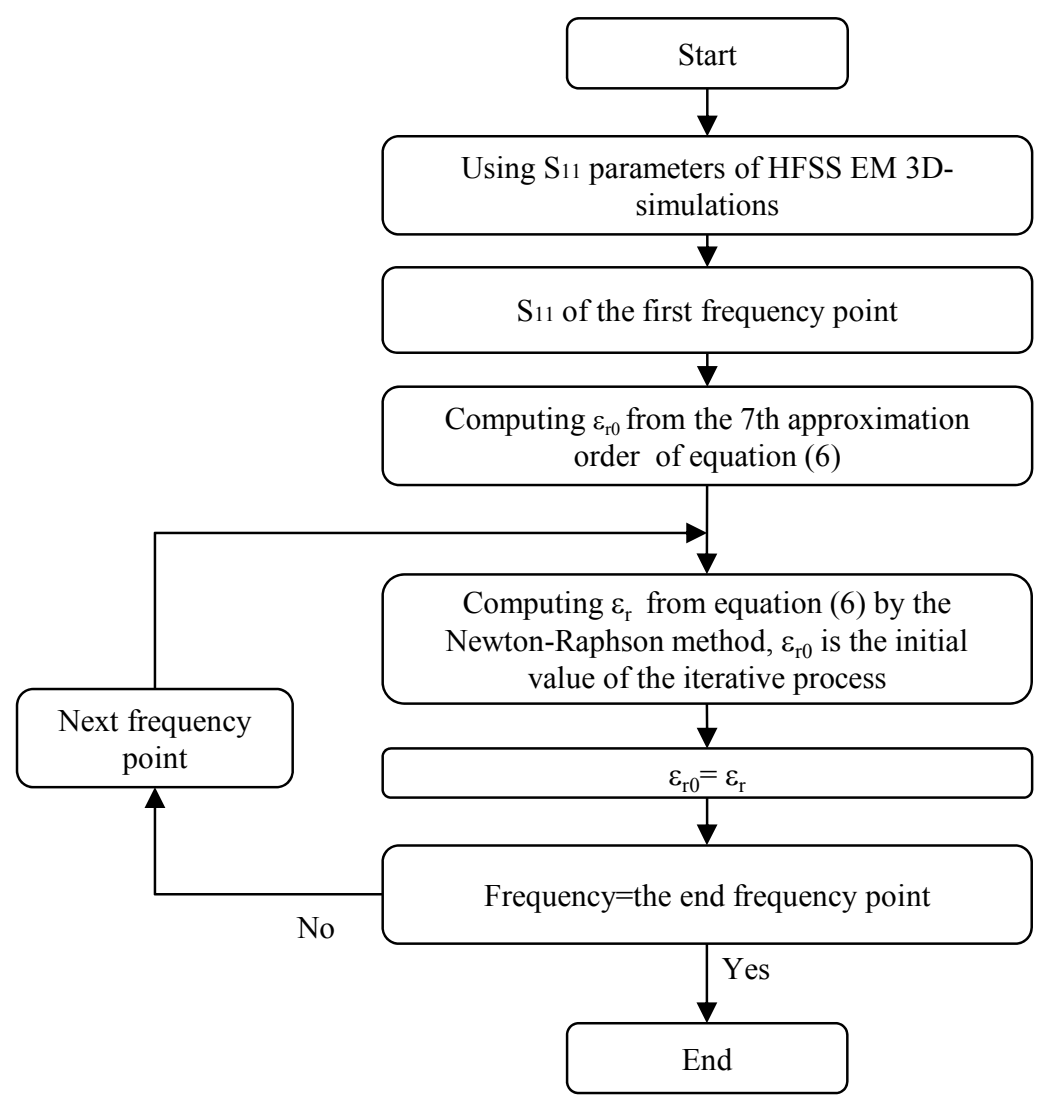

Fig. 5. Flowchart used for complex permittivity calculation. 
The simulation parameters are the following:

- Waveguide WR-90.

- Distance between calibration plane and air-sample interface $l=70 \mathrm{~mm}$.

- Frequency range: [8.2-12.4] GHz (X-band).

- Sample thicknesses: $d=5 \mathrm{~mm}$ for Teflon, $d=2 \mathrm{~mm}$ for wood and $d=1 \mathrm{~mm}$ for distilled water.

- The inner walls of waveguide are assumed perfectly conductor.

The numerical results of relative complex permittivity of Teflon, wood and distilled water are presented graphically in Fig. 6, 7 and 8 respectively. The results of the distilled water are compared with the theoretical Debye model values [3]. And the results of the wood slab are compared with the measured data obtained by the Transmission / Reflection method [8] of $10 \mathrm{~mm}$ wood sample thickness. To do these comparisons, the Debye model and measured data are incorporated in the simulated material properties.

All findings values agree well with the published data [3], [8], [13] and measurements. Generally, the numerical methods require an initial guess to obtain a convergence to the exact permittivity of the material under test. But the results reported in Fig. 6, 7 and 8 are obtained by the initial values $\varepsilon_{\mathrm{r} 0}$. As indicated previously, $\varepsilon_{\mathrm{r} 0}$ is calculated by the $7^{\text {th }}$ A.O. equation. However, we do not need any information about the $\varepsilon_{\mathrm{r}}$ range. It is seen from Fig. 6, 7 and 8 that we can retrieve the relative permittivity of thin or thick simulated materials from their $S_{11}$ parameter. For very low loss materials, the uncertainty of $S_{11}$ increases greatly for very thin samples. The solution might be cutting the sample with a few millimeters thick. For the other extreme case, i.e. for very high loss materials, thick samples might absorb all the electromagnetic wave energy. However, the certainty to determine accurately $\varepsilon_{\mathrm{r}}$ is very low. The solution might be cutting the sample with a thickness less than one millimeter.

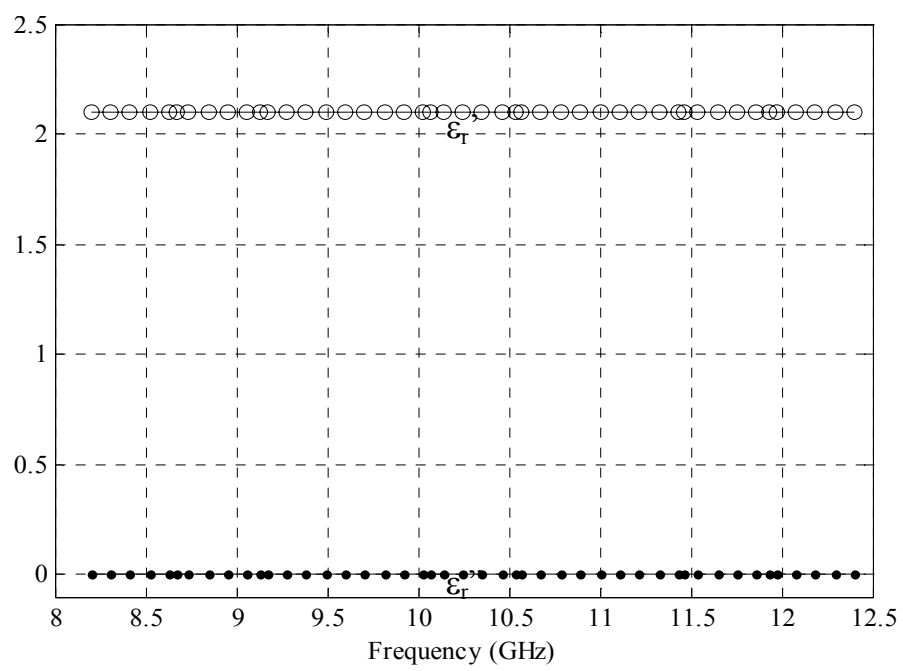

Fig. 6. Simulated relative complex permittivity of $5 \mathrm{~mm}$ long Teflon sample by the proposed method. 


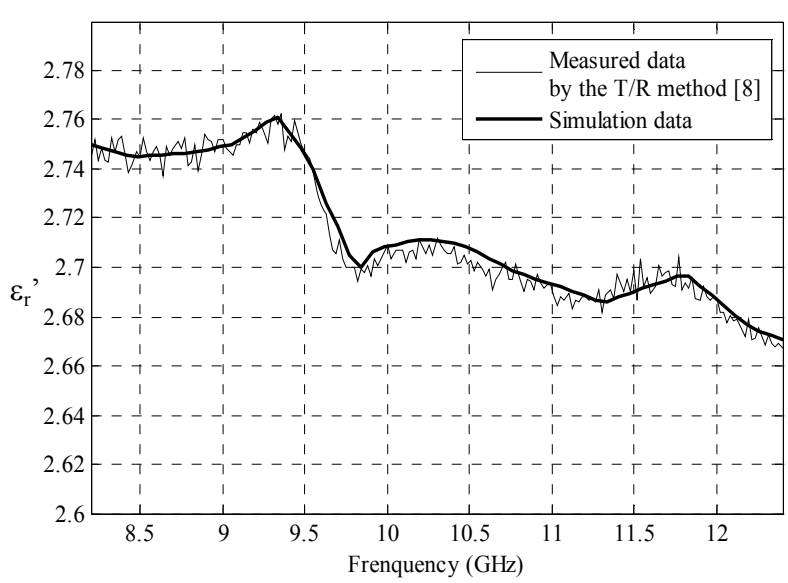

(a)

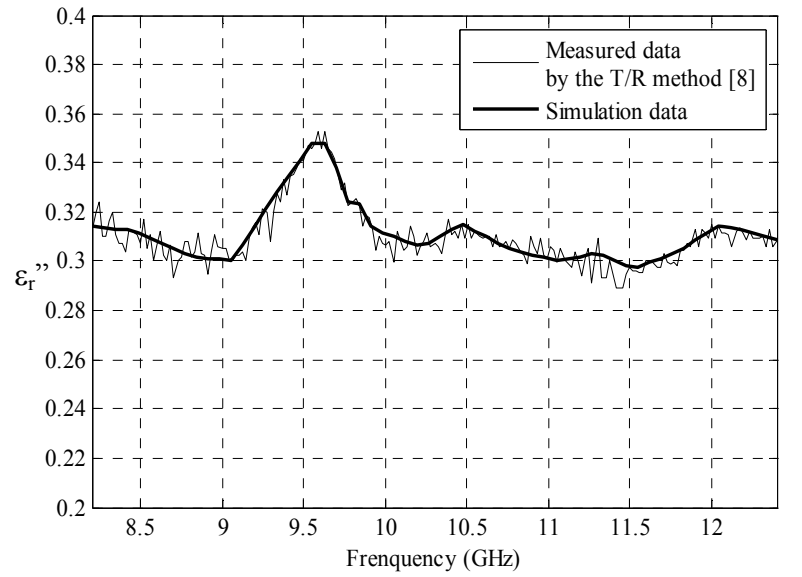

(b)

Fig. 7. (a) and (b) are the real and imaginary parts of the relative complex permittivity of wood sample respectively. The plots represent a comparison between the simulation and the measured results of $2 \mathrm{~mm}$ and $10 \mathrm{~mm}$ wood sample thicknesses respectively. We note that the measured data are obtained by the Transmission / Reflection (T/R) method [8] and incorporated in the simulated material properties. The simulation results are performed by the proposed method.

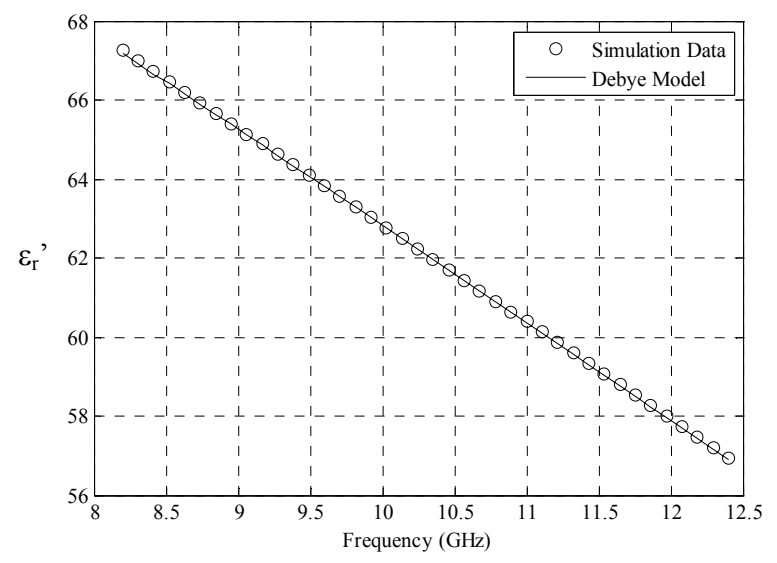

(a)

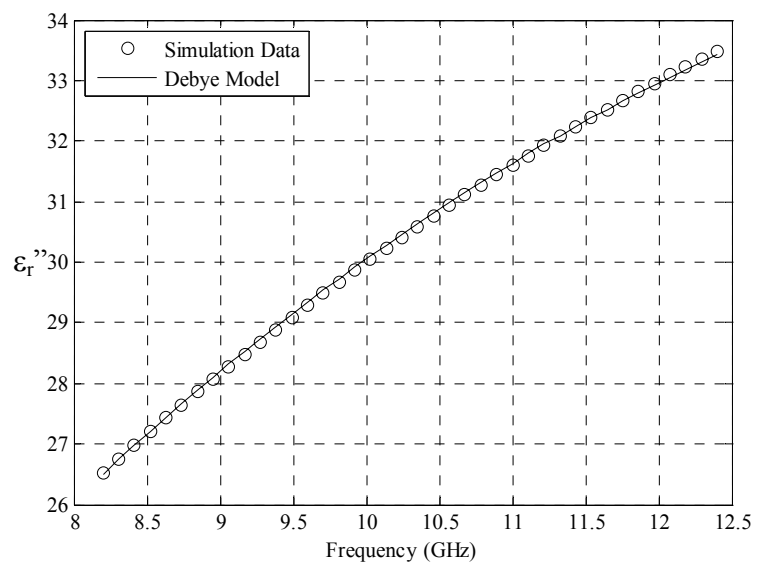

(b)

Fig. 8. Simulated relative complex permittivity ((a) real part and (b) imaginary part) of $1 \mathrm{~mm}$ distilled water thickness by the proposed method. The results are compared with Debye model.

\section{CONCLUSION}

An improved method is developed for complex permittivity determination of thin and moderate thick liquid and solid materials. The samples are in direct contact to the metal plane of the shortcircuited rectangular waveguide without any air gap. The dielectric constant is then calculated from the simulated complex reflection coefficient at the input of the waveguide section. The errors arising from any position offset of the sample are eliminated. The method approximates the hyperbolic tangent terms which produce multiple solutions for complex permittivity determination; and the unique solution that corresponds to the best propagated mode is selected. High accurate solution can be attained by increasing the degree of the approximation. Then, this $\varepsilon_{\mathrm{r}}$-solution is considered as an initial value of the iterative process resolving the exact expression of the reflection coefficient. However, the complex permittivity is determined more accurately. The method is verified numerically 
for Teflon, wood and distilled water. The method can retrieve, with minor errors, the complex permittivity values integrated in simulations. The experimental validation will be performed in the near future.

\section{REFERENCES}

[1] D. Michali, C. Apollo, R. Pastore, and M. Marchetti, "X-Band microwave characterization of carbon-based nanocomposite material, absorption capability comparaison and RAS design simulation," Composites Science and Technology, vol. 70, pp. 400-409, Nov. 2010.

[2] H. Ebara, T. Inoue, and O. Hashimoto, "Measurement method of complex permittivity and permeability for a powdered material using a waveguide in microwave band," Science and Technology of Advanced Materials, vol. 7, pp. 77-83, Feb. 2006.

[3] U. C. Hasar, O. Simsek, M. K. Zateroglu, and A. E. Ekinci, "A microwave method for unique and non-ambiguous permittivity determination of liquid materials from measured uncalibrated scattering parameters," Progress In Electromagnetics Research PIER, vol. 95, pp. 73-85, 2009.

[4] A. Mdarhri, M. Khissi, M. E. Achour, and F. Carmona, "Temperature effect on dielectric properties of carbon black filled epoxy polymer composites," Eur. Phys. J. Appl. Phys., vol. 41, pp. 215-220, Apr. 2008.

[5] L. F. Chen, C. K. Ong, C. P. Neo, V. V. Varadan, and V. K. Varadan, Microwave Electronics Measurement and Material Characterization, John Willey and Sons, West Sussex, England, 2004, 37.

[6] C. P. Rubinguer, and L. C. Costa, "Building a resonant cavity for the measurement of microwave dielectric permittivity of high loss materials," Microwave Opt. Tech. Lett., vol. 49, pp. 1687-1690, July. 2007.

[7] E. Li, Z. P. Nie, G. Guo, Q. Zhang, Z. Li, and F. He, "Broadband measurements of dielectric properties of low-loss materials at high temperatures using circular cavity method," Progress In Electromagnetics Research, PIER, vol. 92, pp. 103-120, 2009.

[8] N. Jebbor, S. Bri, L. Bejjit, A. Nakheli, M. Haddad, and A. Mamouni, "Complex permittivity determination with the transmission / reflection method,” Int. J. Emerg. Sci., vol. 1(4), pp. 682-695, Dec. 2011.

[9] U. C. Hasar, "A fast and accurate amplitude-only transmission-reflection method for complex permittivity determination of lossy materials,"IEEE Transactions on Microwave Theory and Techniques, vol. 56, pp. 2129-2135, 2008.

[10] U. C. Hasar, "Permittivity measurement of thin dielectric materials from reflection-only measurements using one-port vector network analyzers," Progress In Electromagnetics Research, PIER, vol. 95, pp. 365-380, 2009.

[11] U. C. Hasar, and O. Sismek, "An accurate complex permittivity method for thin dielectric materials," Progress In Electromagnetics Research, PIER, vol. 91, pp. 123-138, 2009.

[12] K. Sarabandi, and F. T. Ulaby, "Technique for measuring the dielectric constant of thin materials," IEEE Transactions on Instrumentation and Measurement, vol. 37 pp. 631-636, Dec. 1988.

[13] B. K. Chung, "Dielectric constant measurement for thin materials at microwave frequencies," Progress In Electromagnetics Research, PIER, vol. 75, pp. 239-252, 2007.

[14] J. Baker-Jarvis, Transmission/reflection and short-circuit line permittivity measurements, NIST Note 1341. U.S. Government Printing Office, Washington, D.C., July 1990.

[15] C. A. Balanis, Advanced Engineering Electromagnetics, John Wiley \& Sons, New Jersey, NJ, 1989. 